\title{
EFECTOS OLVIDADOS Y DESARROLLO A ESCALA HUMANA, UNA PROPUESTA ALTERNATIVA PARA LA EVALUACIÓN DE PLANES DE DESARROLLO - CASO USME (BOGOTÁ) 2013-2016
}

\author{
Forgotten Effects and Development on a Human Scale, an Alternative Proposal for the Evaluation \\ of Development Plans - Case: USME (Bogotá) 2013-2016
}

LINDA GIL

Universidad Austral, Chile

\begin{tabular}{|c|c|}
\hline KEY WORDS & ABSTRACT \\
\hline $\begin{array}{l}\text { Development Plan } \\
\text { Human Scale }\end{array}$ & $\begin{array}{l}\text { Between } 2012 \text { and 2016, the Mayor's Office of Bogotá, Colombia, } \\
\text { executed a Development Plan (PD) called "Bogotá Humana". In order to }\end{array}$ \\
\hline Forgotten Effects & alternatively evaluate the incidences of this $P D$ and that local \\
\hline Satisfactory & phenomena with high degrees of complexity were not deformed, \\
\hline Complexity & simplified or separated from reality, the proposal of Development on a \\
\hline Incentive & Human Scale, was used together with the alternative of identification of \\
\hline Behavior & Forgotten Effects. Obtaining in this evaluation, routes with probabilities \\
\hline Public Policy & $\begin{array}{l}\text { of occurrence of forgotten effects on fundamental basic human needs } \\
\text { greater than } 8 \% \text {. The existence of synergy was demonstrated, by } \\
\text { presenting characteristics of multiple incidence. }\end{array}$ \\
\hline
\end{tabular}

PALABRAS CLAVE

Plan de Desarrollo

Escala Humana

Efectos Olvidados

Satisfactor

Complejidad

Incentivo

Comportamiento

Política Pública

\section{RESUMEN}

Entre los años 2012 y 2016 la Alcaldía Mayor de Bogotá, Colombia, ejecutó un Plan de Desarrollo (PD) denominado "Bogotá Humana". Para evaluar de manera alternativa las incidencias de este PD y que fenómenos locales con altos grados de complejidad, no fueran deformados, simplificados o separados de la realidad, se empleó la propuesta de Desarrollo a Escala Humana, unida a la alternativa de identificación de Efectos Olvidados. Obteniendo en esta evaluación, rutas con probabilidades de ocurrencia de efectos olvidados sobre las necesidades humanas básicas fundamentales mayores al 8\%. Se demostró la existencia de sinergia, al presentar características de incidencia múltiple.

Recibido: 10/02/2020

Aceptado: 23/07/2020

\section{GLOBAL KNO KNOWLEDGE}




\section{Introducción}

$\mathrm{E}$ ntre los años 2012 y 2016 en la ciudad de Bogotá, se llevó a cabo el PD Bogotá Humana (PD-BH) consignado en el Acuerdo 489 de 20121. Buscaba tres objetivos; cuidar y defender la vida en el territorio a través del cuidado del agua, la adaptación de la ciudad al cambio climático y la generación de transformaciones sociales (Concejo de Bogotá, 2012). Los objetivos articuladores de la política pública, que pasaron a ser los tres ejes que dieron estructura al PD-BH fueron:

1. Combatir la segregación social y construir una ciudad incluyente, digna y con equidad.

2. Construir una ciudad que no deprede la naturaleza, revitalizada, con espacios públicos y amplia movilidad.

3. Combatir la corrupción y la inseguridad, profundizando la democracia con participación y poder ciudadano.

Estos, se extendieron a lo largo de cada una de las 20 localidades que componen la ciudad de Bogotá, a través de acuerdos locales. De acuerdo al PD-BH, "La visión centrada en el ser humano se aleja de aquella que concibe el desarrollo como una consecuencia unívoca del crecimiento económico. El aumento de la riqueza es una condición necesaria del desarrollo, pero no es suficiente para crear las condiciones que les permita a las personas desplegar todo su potencial como sujetos libres. Hasta donde sea posible, las políticas distritales deben evitar que el crecimiento económico genere desigualdad y segregación." (Alcaldía Mayor, 2011). El objetivo principal del PD-BH, era recudir la segregación social, la discriminación y se pusiera al ser humano en el centro de las preocupaciones del desarrollo. Para los años 2013-2016, Usme una de las 20 localidades, contó de manera particular con un Plan de Desarrollo Económico, Social, Ambiental y de Obras Públicas. Adoptado en un acuerdo local como PD "Usme humana, habitable, participativa y con movilidad social". (Junta Administradora local de Usme, 2012). El cual es el caso de estudio tomado para esta investigación.

\footnotetext{
1 Ver Acuerdo 489 de 2012 en: https://www.shd.gov.co/shd/sites/default/files/documentos/plan _desarrollo_2012_2016.pdf
}

De manera general, los Planes de Desarrollo (PD) son instrumentos de gestión, que permiten de manera planificada y a través de un acuerdo político transparente y claro impulsar el desarrollo en los territorios. En el Título XII, capítulo II, artículo 339 de la Constitución Política de Colombia de 1991, se establece que “[...] las entidades territoriales elaborarán y adoptarán de manera concertada entre ellas y el Gobierno Nacional, planes de desarrollo, con el objeto de asegurar el uso eficiente de sus recursos y el desempeño adecuado de las funciones que les hayan sido asignadas por la Constitución y la ley". Los PD como instrumento formales y legales que trazan objetivos de gobierno, permiten la subsecuente evaluación de su gestión.

Los PD, al estar diseñados para intervenir en las realidades de las comunidades, promueven cambios asociados a grandes problemas de interés público. Al ser instrumentos de gobierno para dar solución a problemas públicos, los PD incidirán en otros problemas públicos que no eran el objeto original de la intervención (Abarca, 2002). Significando esto, que detrás de cada PD existen problemas públicos de difícil solución, lo que complejiza la evaluación real de las incidencias que tendrán las acciones ejecutadas en los PD.

Las evaluaciones se constituyen como mecanismos útiles para medir la eficacia de las intervenciones públicas de los PD, pero implican un gran reto, debido a las características particulares de los diferentes territorios. El alto grado de complejidad en múltiples dimensiones que interactúan de manera simultánea, dificulta la forma en que se abordan los problemas a intervenir. La Secretaría Distrital de Planeación (SDP) en la "Guía para la Formulación, Ejecución, Seguimiento y Evaluación de los Planes de Desarrollo distrital y local", expedida en el año 2011, determina que los efectos e impactos obtenidos en la realidad que se busca transformar con los PD en territorio nacional se realizan con evaluaciones a partir de indicadores que miden las estrategias y los recursos invertidos (Secretaría Distrital de Planeación, 2011:55). Según la SDP, una vez establecidos los objetivos específicos de los PD, y teniendo definidas las principales estrategias en una 
estructura programática, se formulan las metas concretas que miden el avance en el cumplimento de los compromisos establecidos. Las metas pueden ser de tres tipos; de resultado, de producto y de proceso o de gestión. Cada meta, se evalúa a partir de indicadores crecientes, decrecientes, constantes o de suma, según la programación del periodo a medir. Dichos indicadores permiten medir de manera lineal variables relacionadas (Secretaría Distrital de Planeación, 2011).

Sumando a lo anterior se ha de tener en cuenta que los PD al estar relacionados con la intervención a problemas públicos complejos, se convierten en "problemas perversos". Los problemas perversos ${ }^{2}$ son aquellos problemas complejos en los que no hay un buen alineamiento entre costos $y$ valores (Cunningham, 2001). Rittel y Webber (1973) caracterizan de manera general a los problemas perversos como aquellos que no tienen una definición definitiva, son esencialmente únicos y cada problema perverso es un síntoma de otro problema. Con esta descripción todo problema público es en sí mismo un problema perverso.

Así las cosas, se dificulta aún más la correcta evaluación de los efectos generados luego de una intervención pública. Pues existirán siempre lo que Kaufmann y Gil-Aluja (1988) llaman "Efectos Olvidados" (EO). Este tipo de efectos, no siempre son explícitos, visibles o percibidos de manera inmediata, transformándose en "efectos de efectos, de una acumulación de causas" (Kaufmann y Gil- Aluja, 1988:7-8), especialmente cuando se refieren a decisiones políticas, legislativas o económico-sociales. Un PD entonces, tendrá EO a causa de su naturaleza como problema perverso. Entonces, las intervenciones públicas incluyen un alto grado de complejidad y sus efectos no pueden ser simplificados, ni tampoco ser separados de la realidad. Se hace necesario optar por herramientas que faciliten y complementen la visualización de los fenómenos a evaluar. Es por esto que los subconjuntos borrosos, que intentan recoger los fenómenos de manera más precisa y cierta, tal como se presentan en la realidad, identificando efectos de un conjunto de

2 Wicked problems, según la definición original de Rittel y Webber (1973) entidades sobre otro o sobre sí mismo (Kaufmann y Gil-Aluja, 1986), permiten modelar de una mejor manera las incidencias de las intervenciones públicas, con el fin de reflejar la incertidumbre que hay sobre las mismas.

La propuesta de los EO resulta entonces apropiada como herramienta de visualización de la realidad compleja de los efectos directos e indirectos de los PD, ya que permite relacionar la incidencia de causas y efectos en matrices multidimensionales, pues "a través de las matrices de incidencias cualitativas se pueden investigar diversos mecanismos de causa efecto que aún no es posible descubrir a través de la intuición o la experiencia" (Kaufmann y Gil-Aluja, 1986:133).

Al respecto, algunos autores han trabajado la complejidad aplicada a la sostenibilidad. Destacándose estudios sobre; evaluación de la sostenibilidad en la gestión forestal (Ducey y Larson, 1999), medición de la sostenibilidad del desarrollo (Phillis y Andriantiatsaholiaina, 2001) y evaluación de la sostenibilidad en las organizaciones (Muñoz, Rivera, y Moneva, 2008).

En relación a la administración pública, en el año 2012, el modelo de EO se aplicó al desarrollo turístico. El fin de la investigación era conocer las incidencias de las inversiones de la administración pública, sobre el desarrollo turístico de una ciudad, encontrando en primer lugar, las acciones útiles para atraer turismo y en segundo lugar, conocer el efecto multiplicador que generan las inversiones realizadas. Con los resultados obtenidos la administración pública, establecería prioridades en la distribución de los recursos para las acciones de mayor repercusión en el desarrollo económico y de vida (GilLafuente y Kyun Oh, 2012).

EO se aplicó también en la comuna de Valdivia, Chile para medir las incidencias de las políticas públicas para la reducción de la contaminación del aire debido al uso de leña para la calefacción doméstica y sus EO (Manna, Rojas-Mora y Mondaca-Marino, 2018). Entre los principales hallazgos, se encontró que existe una relación indirecta entre el subsidio a la leña de mejor calidad y la mejora de la aislación térmica de los hogares. Invertir en la primera, una solución simple de corto plazo, afectará la 
necesidad de implementar la segunda, una solución compleja de largo plazo.

Adicional a lo anteriormente expuesto, se ha de tener en cuenta que los PD, al intervenir en las comunidades afectan especialmente a las personas y su desarrollo. Es así como los PD deben contemplar siempre un componente relacionado al Desarrollo Humano. Max-Neef, Elizalde y Hopenhayn (1986), plantean una opción abierta para un Desarrollo Humano sistémico a razón de las interacciones existentes entre múltiples factores. Proponiendo, el Desarrollo a Escala Humana (DEH), no como un modelo estandarizado para el desarrollo, sino como un punto de partida para la construcción permanente del mismo. Max-Neef, Elizalde y Hopenhayn, identifican la complejidad de las interacciones del desarrollo cuando dicen "en medio de una realidad actual que nos agobia: «Ya no nos queda ningún hermoso problema particular»", (Max-Neef Elizalde y Hopenhayn, 1986:16). Mencionan que la evidencia central, es que las nuevas calamidades sociales que se manifiestan, ya no se relacionan con problemas específicos, sino como problemáticas complejas que no se pueden seguir abordando satisfactoriamente, mediante la aplicación exclusiva de políticas convencionales inspiradas por disciplinas reduccionistas (Max-Neef Elizalde y Hopenhayn,1986).

El DEH se ha utilizado como base para proponer evaluaciones de políticas de desarrollo. En el año 2009, se presentó una extensión metodológica del $\mathrm{DEH}$, en donde se propone adicionar una matriz proposicional, un indicador de tendencia (positiva o negativa dentro de la situación identificada), con nuevos modos numéricos y gráficos, para presentación de resultados. La representación numérica, consiste en dividir en proporciones iguales cada una de las necesidades existenciales completando un $100 \%$ en su correspondiente necesidad axiológica (Cruz, Atahel, y Max-Neef, 2009).

El DEH, también se utilizó de manera analítica en evaluaciones de impacto de programas institucionales, específicamente en una investigación realizada en la universidad del Quindío en Colombia, en donde se muestra cómo verificar y cuantificar beneficios en el corto plazo y analizar la satisfacción de las necesidades básicas propuestas por el DEH, mediante un procedimiento denominado "Evaluación del Impacto Global". Para la evaluación, se comparan la matriz de condiciones iniciales en un punto cero y una matriz de condiciones esperadas. En ambos casos, definiendo categorías (necesidades axiológicas), variable (atributo susceptible a cambio), condición regular, satisfactor, condición inicial, indicador, nivel de indicador $y$ observación (Sanchez y Ramírez, 2010).

Como se mencionó inicialmente, en la actualidad las evaluaciones del PD se realizan a partir de la medición de metas de resultado, metas de producto y metas de proceso o gestión sin tener en cuenta ni la complejidad de los problemas públicos ni la visión de Desarrollo Humano. Dejando de lado la complejidad real de los impactos y efectos obtenidos con las intervenciones en sitio de los PD. Por lo tanto, se hace necesario evaluar de manera adicional las acciones de los PD a través de herramientas que tengan en cuenta la complejidad global de las intervenciones a los problemas públicos y su interrelación con el desarollo ser humano.

El aporte de esta investigación es la incorporación de una visión sistémica que tiene en cuenta tanto la complejidad de las problemáticas públicas, como el componente de desarrollo humano en las evaluaciones de los PD a nivel local. Lo anterior, mediante una evaluación alternativa y participativa, que identifica la incidencia de acciones propuestas en acuerdos locales, frente a comportamientos esperados a impactar con los PD. Usando EO para obtener resultados de incidencias y realizando un análisis de Necesidades Humanas Básicas Fundamentales a través de DEH. La aplicación de este aporte metodológico se realizó específicamente sobre el "Plan de Desarrollo Económico, Social, Ambiental y de Obras Públicas 2013-2016", en la localidad de Usme, como ejemplo de evaluación a partir de una visión desde la complejidad.

A lo largo de este documento, se exponen las dos propuestas que se tuvieron en cuenta en esta investigación, la metodología utilizada, el procedimiento realizado para el tratamiento de los datos, los resultados obtenidos con la evaluación de los EO y su respectivo análisis a través de la visión del DEH. 


\section{Marco teórico}

\subsection{Desarrollo a escala humana}

Max-Neef, Elizalde y Hopenhayn, (1986), plantean una opción abierta para un desarrollo humano sistémico a razón de las interacciones existentes entre múltiples factores, proponiendo así, el Desarrollo a Escala Humana (DEH). Éste, se sustenta en tres pilares fundamentales: 1. Las necesidades humanas básicas fundamentales, 2 . La auto dependencia y 3. La articulación orgánica de los seres humanos con la naturaleza y la tecnología, lo global con lo local, lo personal con lo social, la planificación con la autonomía y la sociedad civil con el estado. Estos pilares se sustentan sobre una base sólida construida a partir del protagonismo real de las personas, implicando la transformación de la persona objeto en persona-sujeto del desarrollo, como se muestra en la Figura 1. (Max-Neef, Elizalde y Hopenhayn, 1986).

Figura 1: Fundamentos del Desarrollo a Escala Humana.

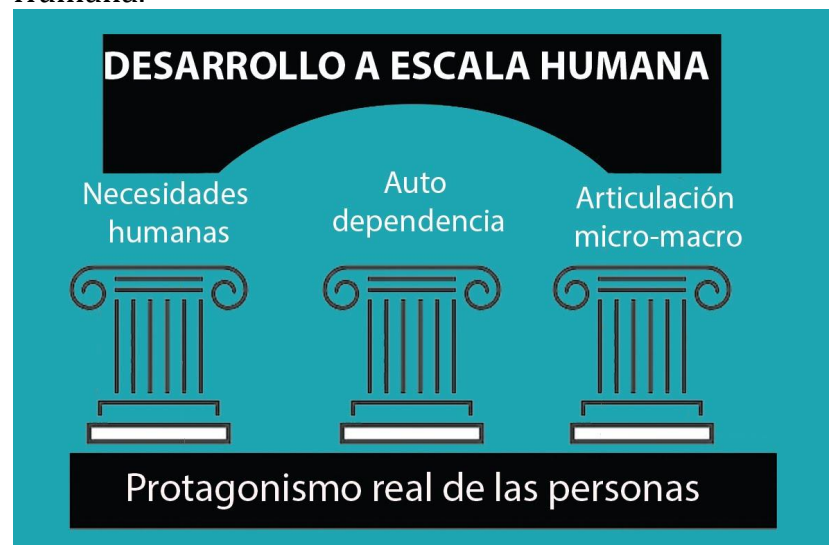

Fuente: Adaptado de Max-Neef, Elizalde y Hopenhayn, 1986.

En relación a las necesidades humanas, el DEH propone que éstas revelan el ser de las personas, ya que este se hace más palpable a través de éstas en su doble condición existencial, como carencia y como potencialidad. No obstante, también han de entenderse como un sistema finito y clasificable, en las que las mismas se interrelacionan e interactúan entre ellas y que pueden desagregarse conforme a múltiples criterios, configurándose en una matriz en la que se cruzan categorías existenciales $\left(\operatorname{ser}^{3}\right.$, tener ${ }^{4}$, hacer ${ }^{5}$ y estar 6 ) y categorías axiológicas (subsistencia, protección, afecto, entendimiento, participación, ocio, creación, identidad y libertad) (Max-Neef, Elizalde y Hopenhayn, 1986). En cada sistema, las necesidades humanas se satisfacen o no se satisfacen a través de la generación o no generación de diferentes tipos de satisfactores propios del territorio. Los satisfactores, según su influencia en el desarrollo humano pueden ser; singulares, sinérgicos, pseudo-satisfactores, inhibidores o destructores.

La relación entre Necesidades Humanas, Satisfactores y Bienes se presenta como se muestra en la Figura 2, "mientras un satisfactor es en sentido último el modo por el cual se expresa una necesidad, los bienes son en sentido estricto el medio por el cual el sujeto potencia los satisfactores para vivir sus necesidades [...] las necesidades humanas fundamentales son atributos esenciales que se relacionan con la evolución; los satisfactores son formas de ser, tener, hacer y estar que se relacionan con estructuras; y los bienes económicos son objetos que se relacionan con coyunturas" (Max-Neef, Elizalde y Hopenhayn,1986:17 ).

Figura 2: Relación entre Necesidades Humanas, Satisfactores y Bienes.

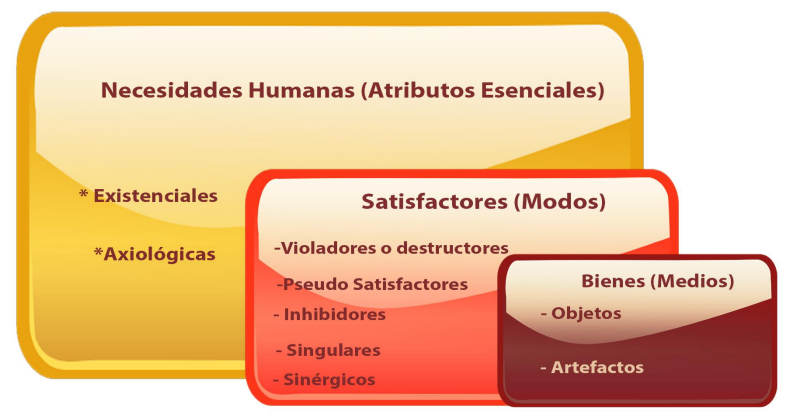

Fuente: Adaptado de Max-Neef, Elizalde y Hopenhayn, 1986.

\subsection{Efectos olvidados}

Los efectos olvidados (EO), son aquellos que no han sido fácilmente identificados en el análisis de

\footnotetext{
${ }^{3}$ Según el DEH, corresponde a sustantivos (atributos personales).

${ }^{4}$ Según el DEH, corresponde a Instituciones, normas, herramientas, mecanismos (no en sentido material).

${ }^{5}$ Según el DEH, corresponde a verbos (acciones).

${ }^{6}$ Según el DEH, corresponde a espacios y/o ambientes (lugares).
} 
expertos, al realizar la relación inicial de causaefecto entre variables (Kaufmann y Gil-Aluja, 1988). Para identificar los EO, se utilizan las matrices de incidencia, que incluyen el mayor número de incidencias entre variables, consideradas como elementos borrosos en una escala de $[0,1]$ dentro de una escala semántica; donde 1 (uno) es verdadero y 0 (cero) es falso. Según Kaufmann y Gil-Aluja, una incidencia se asocia a "la idea de efecto de un conjunto de entidades sobre otro conjunto de entidades o sobre sí mismo" (Kaufmann y Gil Aluja, 1988:13).

La propuesta de EO parte de identificar dos conjuntos finitos de $A$ (Causas) y $B$ (Efectos) así:

$$
\begin{aligned}
& A=\left\{a_{i}, i=1,2 \ldots n\right\} \\
& B=\left\{b_{j}, j=1,2 \ldots m\right\}
\end{aligned}
$$

Existirá una incidencia de $a_{i}$ sobre $b_{j}$ si el valor de la función característica de pertenencia del par $\left(a_{i}, b_{j}\right)$ se haya evaluado en el rango de $[0,1]$, considerando la existencia de la incidencia si el valor es 1 y la no incidencia si el valor es 0 , así:

$$
\mu\left(a_{i}, b_{j}\right) \in[0,1], \forall\left(a_{i}, b_{j}\right) \in A \times B
$$

La evaluación de pares $\left(a_{i}, b_{j}\right)$ entre los conjuntos de $A$ y $B$, definirá la matriz de incidencias directas o efectos de primer orden $A B$ mostrando las relaciones directas de las causas sobre los efectos en diferente grado que se producen entre los elementos del conjunto $A$ y el conjunto $B$ :

$$
A B=\left[\mu\left(a_{i}, b_{j}\right)\right]_{n \times m}
$$

Para la identificación de los EO se deben relacionar los niveles de incidencia que no han sido detectados en la primera matriz. Así, es necesario establecer el hecho de que las causas puedan tener efectos sobre sí mismas y a la vez que los efectos se afecten entre sí mismos. Entonces se han de relacionar causas entre sí, por un lado, y efectos entre sí, por otro. Estas dos matrices auxiliares se definen como:

$$
\begin{aligned}
& A A=\mu\left(a_{i}, a_{j}\right) \in[0,1] \quad i, j=1,2 \ldots \mathrm{n} \\
& B B=\mu\left(b_{i}, b_{j}\right) \in[0,1] \quad i, j=1,2 \ldots \mathrm{m}(6)
\end{aligned}
$$

Tanto la matriz $A A$ como la matriz $B B$, son matrices reflexivas, aunque ni $A A$ ni $B B$ son matrices simétricas. Una vez construidas las tres matrices, se establecen todas las posibles combinaciones de incidencias, tanto directas como indirectas. Para esto se realiza la composición max-min (Kaufmann, 1975,1977):

$$
\begin{aligned}
& A B^{1+2}=A A \circ A B \circ B B \\
& \text { Dónde: } \\
& X \circ Y=\max \min _{j}\left(\mu_{X}(i, j), \mu_{Y}(j, k)\right), \forall i, k
\end{aligned}
$$

Podemos decir que en $\mathrm{AB}^{1+2}$ se acumula los efectos de primero y segundo orden.

La composición max-min es una operación distributiva tal que:

$$
(A A \circ A B) \circ B B=A A(A B \circ B B)
$$

Finalmente, para determinar los efectos de segundo orden, del resultado obtenido se han de sustraer los efectos de primer orden:

$$
A B^{2}=A B^{1+2}-A B
$$

\section{Metodología}

Esta investigación interactúa de manera interconectada entre el componente cualitativo y el cuantitativo a raíz de su naturaleza como problema perverso. El paradigma que enmarca la parte cualitativa de la investigación, es el de la teoría crítica, permitiendo transitar entre la dialógica y la dialéctica obteniendo así la realidad del contexto del entrevistado (Guba y Lincoln, 2002). De manera complementaria, la parte cuantitativa, buscó obtener de manera numérica, relaciones entre múltiples variables a partir de la teoría de los subconjuntos borrosos, permitiendo la clasificación colectiva de incidencias en una escala de valor.

Lo anterior, permitió la recolección de información subjetiva a través de entrevistas semiestructuradas para evaluar el impacto del PD local de Usme, a través de EO y analizar los resultados desde el DEH. A partir de las acciones del PD Local de Usme, se analizaron varios efectos correlacionados.

\subsection{Definición de variables}

Se partió de la premisa general que las políticas públicas impulsan acciones cuyo objetivo es incentivar o desincentivar comportamientos en la población a intervenir. En términos generales 
puede decirse que los incentivos constituyen instrumentos por medio de los cuales se busca afectar el comportamiento de los actores (Jiménez y Podestá, 2009). Así, los conjuntos a evaluar fueron:

- Conjunto Causa: Incentivos claramente definidos en el PD.

- Conjunto Efecto: Comportamientos observables a impactar con el PD.

Para esta investigación se definió como incentivo, aquello diseñado por un PD para fomentar un comportamiento. Los comportamientos observables se definieron como conductas, manera de portarse o actuar.

\subsection{Recolección de datos primarios}

Como primer paso, se realizó una clasificación preliminar de los incentivos y comportamientos claramente definidos en el acuerdo local número 002 del 8 de septiembre de 2012 para el PD local de Usme ${ }^{7}$. Obteniendo así 14 incentivos, 31 comportamientos específicos y 16 comportamientos generales. Con la clasificación obtenida, se realizaron 6 entrevistas semiestructuradas a expertos, basadas en formatos estandarizados ${ }^{8}$ para seleccionar entre los incentivos y comportamientos preliminares, cuales de ellos serían adecuados para ser tenidos en cuenta en las matrices de evaluación aplicadas en el territorio. Estas entrevistas iniciales, se realizaron a personas que trabajaron durante la administración de la $\mathrm{BH}$ en cargos Directivos, Administrativos, Técnicos y Académicos, que tuvieron relación directa con el diseño e implementación del PD-BH durante los años 2012 y 2016.

Tabla 1.

Clasificación de entrevistas iniciales a informantes claves

\begin{tabular}{cc}
\hline Categoría & No Personas \\
\hline Directivos & 1 \\
Administrativos & 2 \\
Técnicos & 2 \\
Académicos & 1 \\
\hline
\end{tabular}

Fuente: Elaboración propia.

\footnotetext{
7 Para mayor información, ver todos los incentivos preliminares hacer clic aquí.

${ }^{8}$ Para mayor información, ver el formato de entrevista utilizado hacer clic aquí.
}

Las entrevistas a expertos, evidenciaron la complejidad del ejercicio de identificación adecuada de incentivos y comportamientos a ser evaluados. Pues la extensión del instrumento de evaluación sería no solo engorrosa para su aplicación, sino tan detallada que podría conducir a errores. Identificada esta dificultad y en pro de no perder el contexto dentro del PD, se decidió para esta investigación, tomar textualmente del objetivo general del acuerdo local, los incentivos que buscaban impactar de manera global comportamientos observables ${ }^{9}$. Seleccionando así 8 incentivos y 10 comportamientos para ser evaluados en el territorio con las matrices de incidencia de EO. Siendo estos los siguientes:

Tabla 2.

Comportamientos e incentivos finales, presentes en el objetivo general del acuerdo local

\begin{tabular}{|c|c|}
\hline Comportamientos & Incentivos \\
\hline $\begin{array}{l}\text { 1. Segregación } \\
\text { social, económica, } \\
\text { espacial y cultural }\end{array}$ & $\begin{array}{l}\text { 1. Atención integral a } \\
\text { niños y niñas de } 0 \text { a } 5 \text { años. }\end{array}$ \\
\hline $\begin{array}{l}2 . \quad \text { Calidad de vida } \\
\text { del conjunto de la } \\
\text { población local }\end{array}$ & $\begin{array}{l}2 . \quad \text { Acciones } \\
\text { enfocadas a la formación } \\
\text { de ciudadanas y } \\
\text { ciudadanos jóvenes activos } \\
\text { y capaces de transformar } \\
\text { el tejido social barrial, } \\
\text { local, distrital, nacional e } \\
\text { internacional }\end{array}$ \\
\hline $\begin{array}{l}3 . \quad \text { Articulación a } \\
\text { una sociedad } \\
\text { posmoderna en el marco } \\
\text { de condiciones de } \\
\text { seguridad y convivencia. }\end{array}$ & $\begin{array}{l}3 . \quad \text { Implementación } \\
\text { de un modelo de } \\
\text { ordenamiento local } \\
\text { alrededor del agua }\end{array}$ \\
\hline $\begin{array}{l}\text { 4. Efectos del } \\
\text { cambio climático }\end{array}$ & $\begin{array}{l}4 . \quad \text { Impulsar los } \\
\text { pobladores rurales como } \\
\text { actores fundamentales en } \\
\text { la organización socio- } \\
\text { espacial, articuladores de } \\
\text { la región-capital y } \\
\text { constructores del borde } \\
\text { urbano-rural del distrito. }\end{array}$ \\
\hline $\begin{array}{l}\text { 5. Generación de } \\
\text { residuos }\end{array}$ & $\begin{array}{l}\text { 5. Fortalecimiento de } \\
\text { lo público, la participación } \\
\text { y veeduría ciudadana }\end{array}$ \\
\hline $\begin{array}{l}\text { 6. Consolidación } \\
\text { progresiva de la } \\
\text { identidad Usmeña. }\end{array}$ & $\begin{array}{l}\text { 6. Modelo de gestión } \\
\text { pública orientado a la } \\
\text { productividad desde la }\end{array}$ \\
\hline
\end{tabular}

${ }^{9}$ Para mayor información, ver el objetivo del acuerdo local con incentivos y comportamientos hacer clic aquí. 


\begin{tabular}{|c|c|}
\hline & $\begin{array}{l}\text { creatividad, diversidad } \\
\text { cultural, el diálogo } \\
\text { intercultural y de saberes. }\end{array}$ \\
\hline $\begin{array}{l}7 . \quad \text { Habitabilidad } \\
\text { territorial para superar } \\
\text { la segregación }\end{array}$ & $\begin{array}{l}7 . \quad \text { Proyectos que } \\
\text { vinculen a la población al } \\
\text { ejercicio de los derechos }\end{array}$ \\
\hline $\begin{array}{l}8 . \quad \text { Salud como } \\
\text { derecho fundamental. }\end{array}$ & $\begin{array}{l}\text { 8. Estrategias que } \\
\text { permitan aliviar la carga } \\
\text { de gasto de los sectores } \\
\text { más pobres de la localidad. }\end{array}$ \\
\hline $\begin{array}{l}9 . \quad \text { Desarrollo } \\
\text { económico popular, } \\
\text { campesino, de redes } \\
\text { locales de producción } \\
\text { agropecuaria y } \\
\text { comercialización }\end{array}$ & \\
\hline $\begin{array}{l}10 . \quad \text { Dependencia } \\
\text { económica social }\end{array}$ & \\
\hline
\end{tabular}

Fuente: Adaptado del local número 002 del 8 de septiembre de 2012 para el PD local de Usme.

Una vez identificados los incentivos y comportamientos a evaluar, esta investigación se centró en recolectar información primaria en las matrices de EO a partir de entrevistas con los actores sociales relacionados con el PD local de Usme. A raíz de la naturaleza dinámica de la investigación, para este caso específico se usó un tipo de entrevista semi-estructurada 10 , que permitió identificar las incidencias de las variables causa-efecto. Las entrevistas, tuvieron como tema central la incidencia de incentivos del PD local, sobre los comportamientos esperados.

\section{Evaluación}

Se construyeron las tres matrices de evaluación $A B(8 \times 10), A A(8 \times 8)$ y $B B(10 \times 10)$ y se aplicaron individualmente a los siguientes actores locales según los roles definidos en la siguiente tabla durante el periodo del PD-BH en la localidad de Usme:

Tabla 3.

Relación de actores locales entrevistados

\begin{tabular}{lc}
\hline \multicolumn{1}{c}{ Rol } & No Personas \\
\hline Planificador local del PD-BH en Usme & 2 \\
Ejecutor local del PD-BH en Usme & 2 \\
Habitante de la Localidad de Usme. & 6 \\
(Receptor o beneficiario del PD-BH) & \\
\hline
\end{tabular}

Fuente: Elaboración propia.

\footnotetext{
10 Para mayor información, ver el formato de entrevista utilizado hacer clic aquí.
}

La información obtenida por persona recolectó 244 respuestas. Para 10 personas se obtuvieron 2440 respuestas, mostrando como resultado inicial los efectos que presentan una relación directa y visible entre las variables de las matrices. Para cada cruce entre fila y columna en cada una de las matrices, se construyó una distribución empírica a partir de las respuestas reportadas por los entrevistados. A continuación se presenta según los datos oficiales de censo de la población y extensión de la localidad de Usme:

Tabla 4.

Características generales Localidad de Usme

\begin{tabular}{ll}
\hline Territorio: & 119.04 Km cuadrados \\
Población (2005): & 426.176 Habitantes \\
\hline
\end{tabular}

Fuente: Adaptado de

http://www.usme.gov.co/content/historia-lalocalidad-5a-usme

Al ser esta una investigación particular y no contar con financiación, no era viable realizar las 384 entrevistas que definía la muestra con un margen de error del $5 \%$ y un nivel de confianza del 95\%. Reconociendo esta limitante y para garantizar que el ejercicio de esta investigación fuera realizable $y$ tuviera una muestra representativa, (según el reporte de habitantes) para esta investigación fue necesario realizar un experimento de Monte Carlo para simular 10.000 réplicas o en este caso simular 10.000 actores. En cada réplica se seleccionó un valor aleatorio de la distribución empírica para cada cruce entre filacolumna de cada una de las matrices presentando efectos de segundo orden al repetir la iteración. Por tanto, los EO obtenidos tienen un carácter estocástico, al aparecer en algunas réplicas, mientras en otras no.

\subsection{Sistematización y análisis de la información}

La información primaria obtenida, permitió la construcción colectiva de las matrices cuantitativas de E011. A través del algoritmo presentado en la Sección 2.2 de este documento, se obtuvieron los EO del PD Local de Usme. Con estos, se realizó un análisis a partir de DEH, identificando y analizando los tipos de

\footnotetext{
11 Para mayor información, ver las matrices utilizadas hacer clic aquí.
} 
satisfactores existentes en el PD local relacionados con las necesidades humanas básicas fundamentales presentes. Finalmente, se validaron los resultados de la investigación con los actores involucrados, realizando entrevistas a profundidad dirigidas hacia la búsqueda de un análisis más riguroso de las opiniones de los actores y hacia la comprensión de las perspectivas que tienen los informantes respecto de la incidencia de los efectos tal como las expresan con sus propias palabras (Flores, 2009).

\section{Resultados}

Con el experimento de Monte Carlo y de las 10.000 réplicas se obtuvieron un total de $\mathbf{1 0 9 8}$ rutas con probabilidades de ocurrencia de entre el $0,0002 \%$ y el $11.4 \%$. Específicamente se encontraron 616 rutas ${ }^{12}$ con efectos de segundo orden desde $A$ hacia $B$ a través de $B$ y 482 rutas $^{13}$ con efectos de segundo orden desde $A$ hacia $B$ a través de $A$. Las rutas de EO obtenidas con más de $8 \%$ de ocurrencia se relacionan a continuación y serán las analizadas a lo largo de esta investigación.

12 Para mayor información, ver los resultados obtenidos $\mathrm{AB}$ por $\mathrm{B}$, hacer clic aquí.

13 Para mayor información, ver los resultados obtenidos AB por A, hacer clic aquí. 
Tabla 5.

E0 desde A hacia B a través de B y desde A hacia B y a través de A, con probabilidad de ocurrencia mayor al 8\%

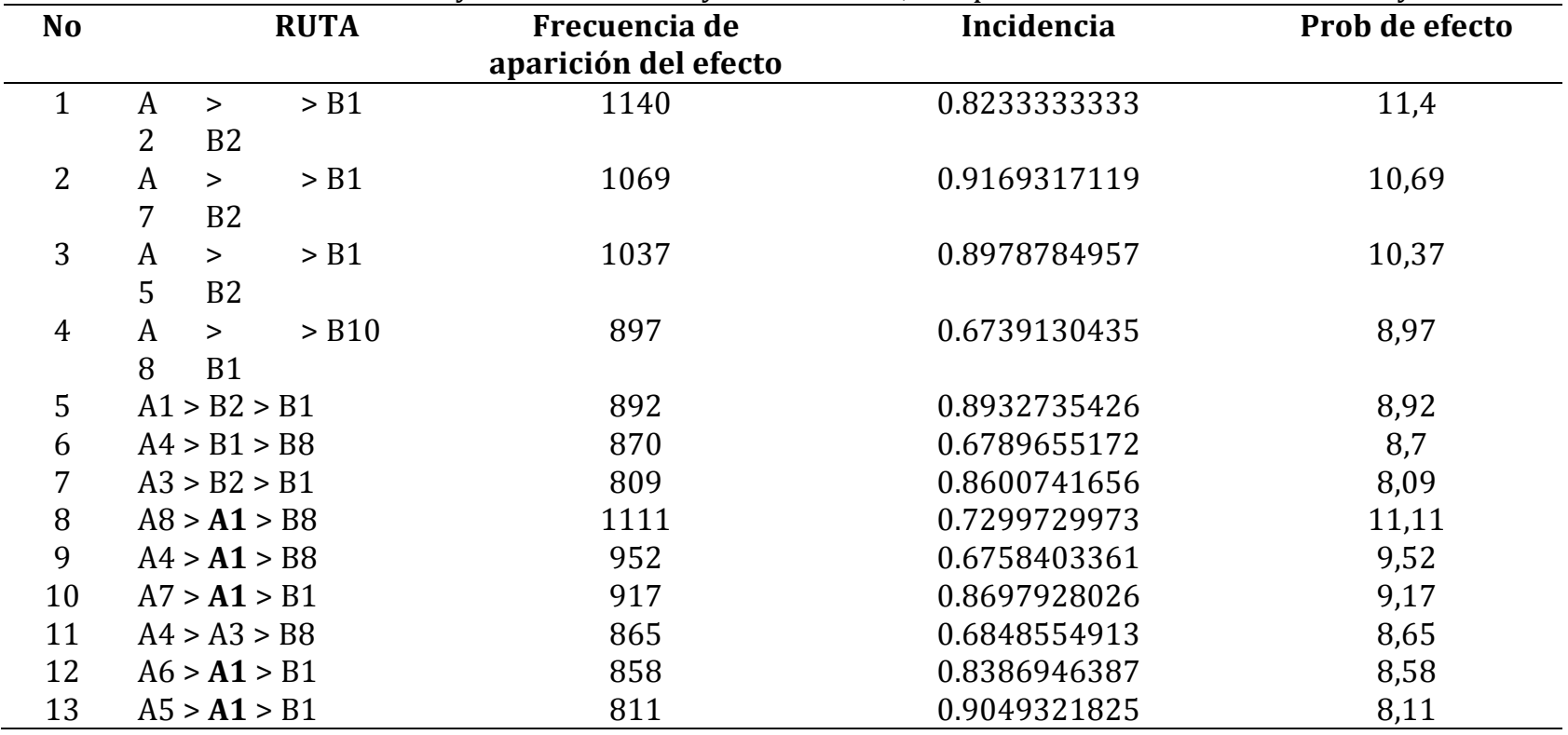

Fuente: Elaboración propia.

De la tabla anterior se identifican trece rutas con tres EO que se repiten reiterativamente, para mayor claridad estas se muestran gráficamente en la Figura 3.

Figura 3. Rutas con los EO con probabilidades mayores al 8\%.

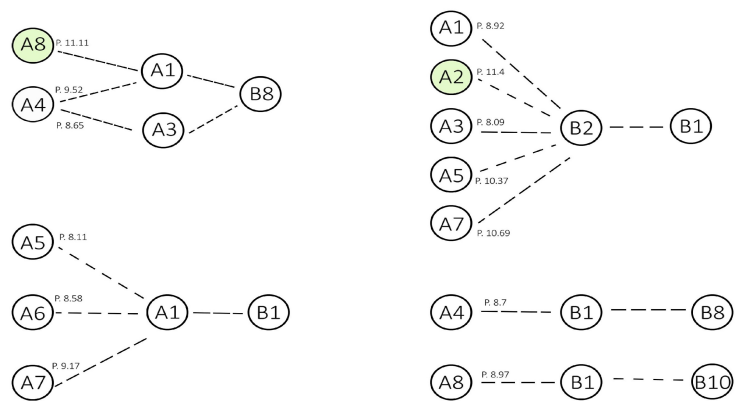

Fuente: Elaboración propia.

De la relación de rutas representadas gráficamente, los dos EO con mayor recurrencia para probabilidades superiores al $8 \%$ son:

- B1: Segregación social, económica, espacial y cultural.

- B8: Salud como derecho fundamental

Los resultados obtenidos con EO, se validaron con los mismos actores inicialmente encuestados que participaron en la investigación a través de entrevistas a profundidad, ${ }^{14}$ para identificar las necesidades básicas fundamentales impactadas con los incentivos y obtener la clasificación propuesta por el DEH de satisfactores descrita en la sección 2.1 de este documento. De tal manera que los incentivos acordados dentro del territorio en el PD local, corresponden en DEH a los satisfactores, al ser modos por los cual se expresan las necesidades de los sujetos. Así, los satisfactores/incentivos del PD se relacionaron con las necesidades humanas según lo consignado en el acuerdo local, como se muestra en la matriz de DEH específica para el PD-BH en la localidad de Usme de la Tabla 6.

Tabla 6.

Matriz de necesidades y satisfactores del PD Local de Usme

\begin{tabular}{ccccc}
\hline Necesidades & Ser & Tener & Hacer & Estar \\
\hline Subsistencia & & A8 & A1 & \\
Protección & & A1, A3 & \\
Afecto & & & \\
Entendimiento & & A2 & \\
Participación & A6, A7 & A2, A4, A5 & A7 \\
Ocio & & & \\
Creación & A6 & A2, A4 & \\
Identidad & A6 & A2, A4 & \\
Libertad & A7 & & A7
\end{tabular}

Fuente: Resultado de la investigación.

\footnotetext{
14 Para mayor información, ver Validación de satisfactores por los actores participantes aquí.
} 
Según la relación de los satisfactores en el PD en la matriz anterior de DEH, se clasificaron las necesidades humanas básicas fundamentales en impactadas y no impactadas desde el PD-BH como se muestra en la Tabla 7.

Tabla 7.

Impacto del PD en las Necesidades Humanas

\begin{tabular}{|c|c|}
\hline Necesidades Impactadas & $\begin{array}{c}\text { Necesidades } \\
\text { No Impactadas } \\
\end{array}$ \\
\hline $\begin{array}{l}\text { Existenciales: } \\
\text { Hacer: } 5 \text { satisfactores (A1, } \\
\text { A2, A3, A4, A5) } \\
\text { Tener: } 3 \text { satisfactores (A6, } \\
\text { A7, A8) } \\
\text { Estar: } 1 \text { satisfactor (A7) } \\
\text { Axiológicas: } \\
\text { Subsistencia: } 2 \text { satisfactores } \\
\text { (A1, A8) } \\
\text { Protección: } 2 \text { satisfactores } \\
\text { (A1, A3) } \\
\text { Entendimiento: } 1 \text { satisfactor } \\
\text { (A2) } \\
\text { Participación: } 5 \text { satisfactores } \\
\text { (A2, A4, A5, A6, A7) } \\
\text { Creación: } 3 \text { satisfactores (A2, } \\
\text { A4, A6) } \\
\text { Identidad: } 3 \text { satisfactores } \\
\text { (A2, A4, A6) } \\
\text { Libertad: } 1 \text { satisfactor (A7) }\end{array}$ & \begin{tabular}{lc}
\multicolumn{2}{c}{ Existenciales: } \\
$-\quad$ Ser \\
Axiológicas: \\
- & Ocio \\
- & Afecto
\end{tabular} \\
\hline
\end{tabular}

Fuente: Elaboración propia.

Sobre la matriz de DEH del PD (Tabla 6), se procedió a trazar las rutas encontradas con los resultados de EO (Figura 3). Se graficaron entonces las interacciones de satisfactores sobre sí mismos y su correspondiente EO encontrado (Figuras 4,5,6 y 7).

En la Figura 4 se muestran las rutas de $A$ hacia $B 8$ a través de $A$ con probabilidades mayores al $8 \%$.
Figura 4. Rutas de A hacía B8 a través de A con los EO con probabilidades mayores al $8 \%$.

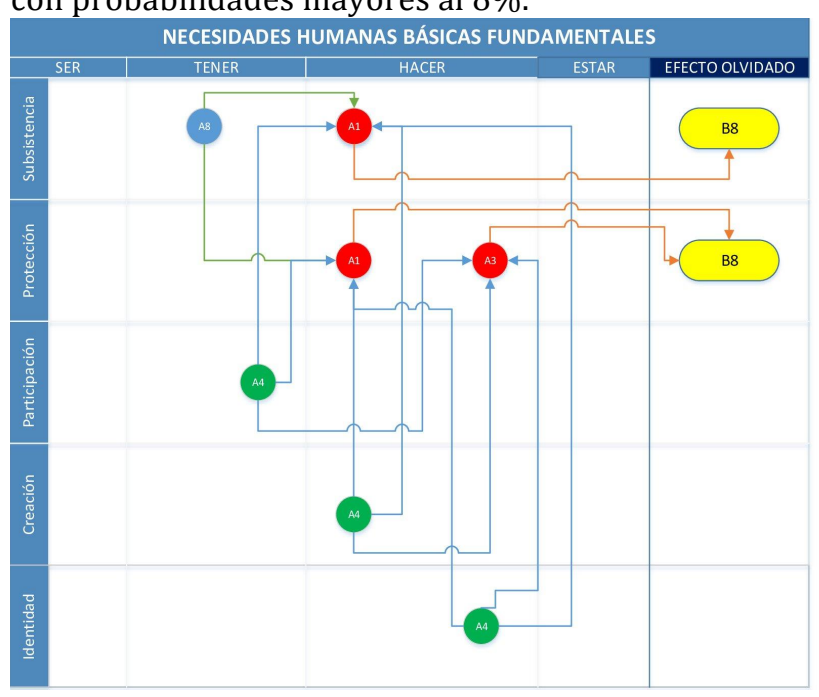

Fuente: Elaboración propia.

De la misma manera se graficaron las rutas de $A$ hacía $B 1$ a través de $A$ con probabilidades mayores al $8 \%$.

Figura 5. Rutas de A hacía B1 a través de A con los EO con probabilidades mayores al $8 \%$.

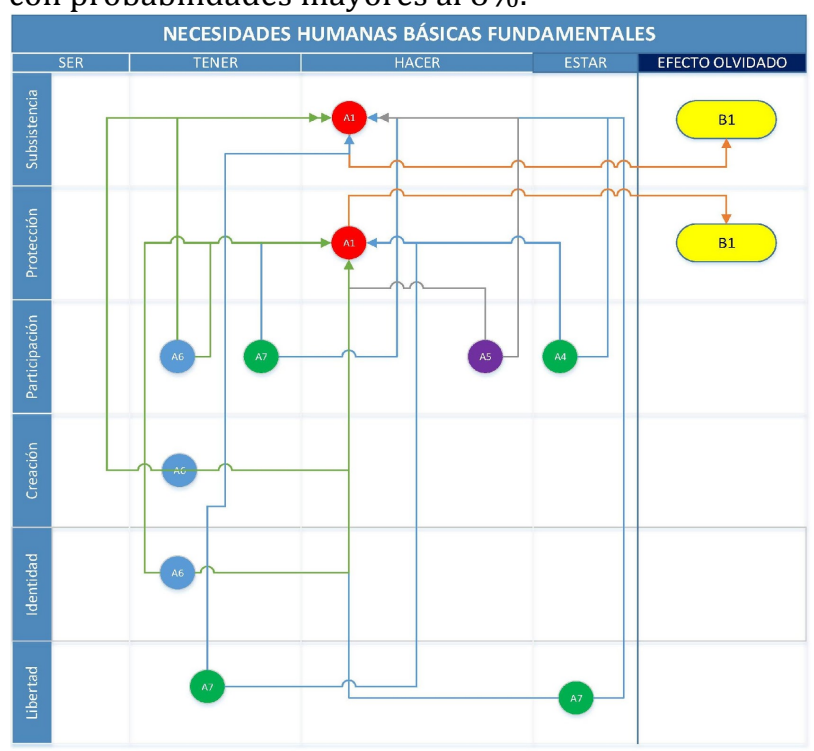

Fuente: Elaboración propia.

Por otro lado, como se muestra en la Figura 6, el satisfactor dentro del PD que no presentó rutas de incidencia con probabilidad mayor de $8 \%$ fue $\mathrm{A} 2$. 
Figura 6. Satisfactor sin incidencia sobre otros satisfactores.

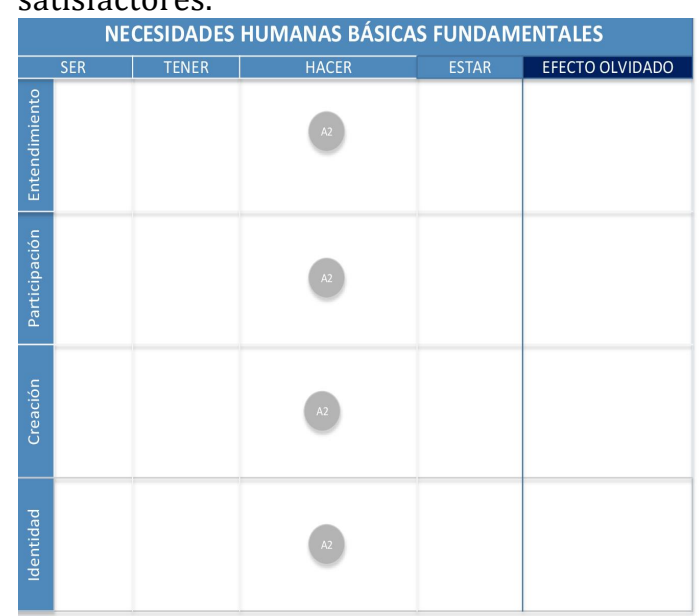

Fuente: Elaboración propia.

Cada una de las figuras anteriores, presenta de manera gráfica y sencilla la relación de EO y $\mathrm{DEH}$, que permite relacionar los satisfactores asociados a necesidades humanas básicas fundamentales y su incidencia en comportamientos observables, dentro del PD-BH específicamente para la Localidad de Usme.

Finalmente, la relación total entre los resultados de los EO y el DEH graficados en la matriz de necesidades humanas se presentan en la Figura 7.

Figura 7: Relación EO con DEH.

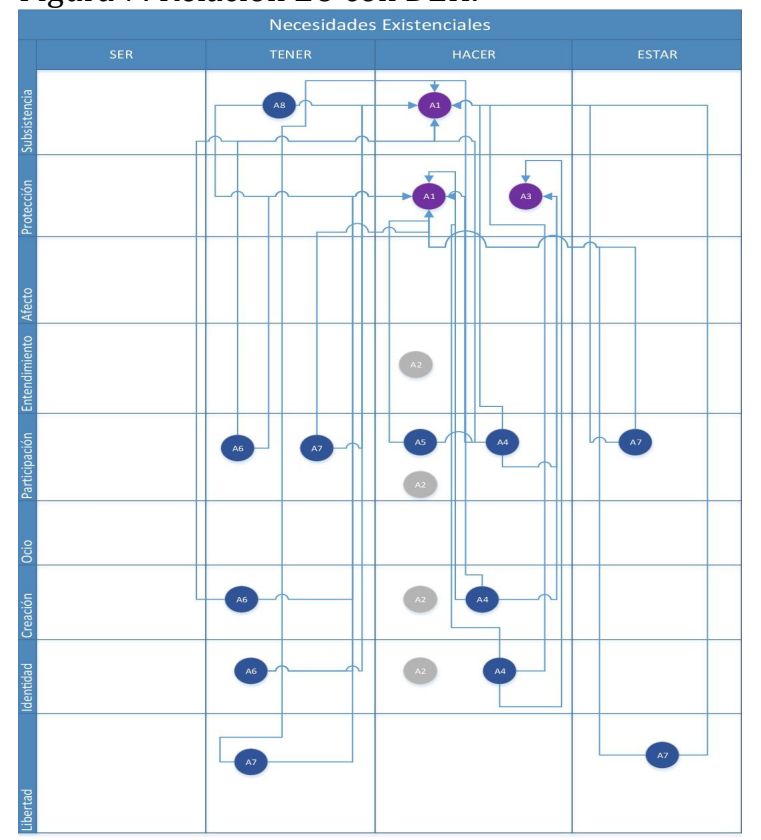

Fuente: Elaboración propia.

\section{Análisis de resultados}

La validación de los resultados encontrado en esta investigación con los actores entrevistados, permitió que los incentivos/satisfactores se clasificaron según su incidencia presentada en EO como se muestra en la Tabla 8. Para ver la validación detallada con los actores remitirse a "Validación de satisfactores por parte de los actores participantes" 15 .
15 Para mayor información, ver Validación de satisfactores por los actores participantes aquí. 
Tabla 6.

Validación y clasificación de satisfactores

\begin{tabular}{cc}
\hline Incentivo/Satisfactor & $\begin{array}{c}\text { Tipo de } \\
\text { satisfactor } \\
\text { según DEF }\end{array}$ \\
\hline
\end{tabular}

A1: Atención integral a Sinérgico

niños de 0 a 5 años

Sinérgico

A través de este satisfactor se
evidencia la generación de
incidencias
múltiples.

Impactando varias necesidades

de manera simultánea.

Tipo de satisfactor Tipo Final según resultados EO

Altamente Sinérgico,
al ser un nodo a
través del cual se
generan los efectos
olvidados B1 y B2.

A2: Acciones enfocadas a la

Sinérgico

El impacto de este satisfactor no

Singular

Singular

formación de ciudadanas y

ciudadanos jóvenes activos

demuestra incidencia sobre

otros satisfactores.

y capaces de transformar el

tejido social, barrial, local

distrital, nacional e

internacional.

A3: Implementación
un modelo
ordenamiento de
alrededor del agua.

Singular

A través de este satisfactor se

evidencia la generación de incidencias múltiples. Impactando varias necesidades de manera simultánea.

Altamente Sinérgico,

al ser un nodo a

través del cual se

generan los efectos olvidados B1 y B2.

A4: Impulsar a los pobladores rurales como

Sinérgico A partir de este satisfactor se evidencia la generación de Sinérgico

Sinérgico actores fundamentales en la organización socioespacial, articuladores de la región-capital y constructores del borde urbano-rural del distrito.

A5: Fortalecimiento de lo público, la participación y veeduría ciudadana

$\begin{array}{clr}\text { Pseudo } & \text { En la validación se identificó } \\ \text { satisfactor } & \text { que no existe una participación } \\ & \text { activa de la comunidad, pues } \\ & \text { quienes participan son } \\ & \text { generalmente los líderes con } \\ & \text { intereses políticos. }\end{array}$
incidencias múltiples sobre otros satisfactores.

$\begin{array}{llll}\text { Sinérgico } & \text { A partir de este satisfactor se } & \text { Sinérgico } & \text { Sinérgico } \\ & \text { evidencia la generación de } & & \\ & \text { incidencias múltiples sobre } & & \\ & \text { otros satisfactores. } & & \end{array}$

A6: Modelo de gestión pública orientado a la productividad, diversidad cultural, el diálogo intercultural y de saberes. A7: Proyectos que vinculen a la población al ejercicio de los derechos
Destructor En la validación se encontró que los proyectos son impuestos por el gobierno y no generados por la comunidad. Se clasifica A7 como destructor al no tener en cuenta las problemáticas particulares del territorio y afectar así el desarrollo de los pobladores.

\begin{tabular}{llll}
\hline A8: Estrategias que & Inhibidor & A8 se clasifica como inhibidor al & Sinérgico \\
permitan aliviar la carga & & afectar negativamente a otras & \\
de gasto de los sectores & necesidades que no eran & \\
más pobres de la localidad. & originalmente tenidas en cuenta. & \\
\hline
\end{tabular}

Fuente: Elaboración propia. 
Los resultados de esta evaluación evidencian que el PD se enfocó mayoritariamente en la satisfacción de la necesidad axiológica de Participación, dejando sin satisfacer las necesidades del Afecto y del Ocio. A su vez quedó sin incentivos/satisfactores la necesidad existencial del ser.

Los incentivos propuestos por el PD según los resultados obtenidos mostraron enfocarse principalmente en la satisfacción del hacer. Las necesidades impactadas que presentaron los EO con mayor recurrencia fueron:

- Existenciales: Hacer

- Axiológicas: Subsistencia, Protección.

Se evidenció la relación de EO y DEH en la evaluación realizada. Se encontró que la satisfacción de las necesidades de participación, libertad y creación a través de las necesidades de subsistencia y protección inciden en la segregación social, económica y espacial, y en la Salud como derecho fundamental.

Como resultado general se evidencia la naturaleza de los satisfactores, demostrando que la sinergia de estos, se da tanto en la necesidad, como en otros satisfactores al presentar características de incidencia múltiple, permitiendo reconocer nuevas características de los satisfactores según los resultados de EO, así:
Tabla 7.

Nuevas características de los satisfactores

No Resultado de Características evidenciada evaluación del satisfactor

1 Satisfactor Que su sinergia inicial es Altamente demostrada a través de sinérgico resultados y su característica (A1, A3) sinérgica se da también a través de sí en otras necesidades no identificadas de manera inicial, como resultado de la interacción con otros satisfactores. (Nodo Intermedio)

2 Satisfactor Que su sinergia inicial es Sinérgico demostrada a través de Simple resultados y su característica (A6) sinérgica genera influencia en la interacción con un satisfactor. (Nodo Inicial)

3 Satisfactor Que su sinergia inicial es Sinérgico demostrada a través de Múltiple resultados y su característica (A4) sinérgica genera influencia en la interacción con dos o más satisfactores. (Nodo Inicial)

4 Satisfactor Que su sinergia inicial no Sinérgico muestra relación con otros Singular satisfactores a través de los (A2) resultados obtenidos, pero sí con otras necesidades. (Nodo sin incidencias)

5 Destructor Que presenta una característica sinérgico inicial como destructor y a (A7) través de resultados muestra características sinérgicas en la interacción con otros satisfactores.

6 Inhibidor Que presenta una sinérgico característica inicial como (A8) inhibidor y a través de resultados muestra características sinérgicas de la interacción con otros satisfactores. (Nodo Inicial)

7 Pseudo Que presenta una Satisfactor con característica inicial como sinergia pseudo satisfactor y a través de negativa (A5) resultados muestra características sinérgicas de la interacción con otros satisfactores. (Nodo Inicial)

Fuente: Elaboración propia. 
De la validación de los resultados de la evaluación con los actores se resalta específicamente para el territorio que:

\begin{abstract}
Usme es una localidad importante porque muestra un conflicto de gran peso, no solo por ser crucial en la ruralidad para Bogotá sino porque conecta a toda la ciudad con el páramo del Sumapaz. Los bordes con indígenas, el tema del agua, las especulaciones sobre el suelo y la frontera agrícola son de los problemas más significativos. Haciendo esto que el problema público que se desea abordar desde el PD sea muy complejo.
\end{abstract}

EL PD apostaba a la implementación de un modelo de ordenamiento alrededor del agua, buscando elevar las capacidades de decisión, de participación, de monitoreo y de vigilancia por parte de los pobladores. Al ser el agua un elemento crítico genera conflicto o genera potencialidades para la gestión comunitaria. La relación entre los pobladores rurales y la implementación de un modelo de ordenamiento territorial alrededor del agua tiene mucha fuerza, porque el agua brinda condiciones para poder decidir sobre el territorio ya que está ligada a la salud de todos los pobladores del territorio. En relación a esto último han existido avances, pero muy pocos porque la mayoría son frenados por interés privados.

La población rural es escasa al igual que su fuerza política. Los intereses están más enfocados en la construcción de viviendas sobre terrenos campesinos para generación de utilidades a la industria de la construcción, que en la generación de proyectos de fortalecimiento del campo. Estos últimos tendrían en el mediano y largo plazo una repercusión positiva al mejorar la calidad de vida para el conjunto de la población, propiciando un ambiente más limpio y menos contaminado.

Aunque no se han presentado muchos proyectos sociales, se están viendo más programas en este sector. Los más significativos son los culturales concentrados especialmente en la zona urbana. A nivel rural faltan muchos más eventos que permitan al campesino ser actor de su propio desarrollo. Al año se hacen dos o tres eventos como mucho. Los eventos grandes generalmente son las "Fiestas del campesino", que son las actividades clásicas celebradas año tras año.

Es necesario romper la idea generalizada en la población que el campo significa atraso y de que las decisiones fundamentales no las pueden tomar los propios habitantes del territorio. Con los cambios en los imaginarios de la gente, se cambian las prácticas que impactarán en condiciones de equidad y a un mayor plazo se generará una mejor calidad de vida del conjunto de la población local. Los proyectos impuestos por gobiernos de turno que cambian cada 4 años no permiten el desarrollo real del territorio porque no permean las problemáticas existentes.

Por otro lado, las estrategias que tiene que ver con el alivio de la carga de los sectores más pobres de la localidad están asociados a subsidios. Esta medida es bastante crítica, pues en algunos casos genera más dependencia y desequilibrios, propiciando aún más la segregación existente. Los subsidios mal planeados no llegan a las personas en condiciones de igualdad, terminan siendo un efecto negativo porque las personas terminan dependiendo del gobierno y no de ellos mismos. La incidencia en la dependencia económica es muy clara. Muchas personas se vuelven dependientes económicamente del Distrito, porque saben que tendrán ayuda sin esfuerzo. Por otro lado, la población de primera infancia requiere subsidios fuertes, ya que es una población muy vulnerable en este territorio. Es en la primera infancia, donde se estimulan capacidades y se generan aprendizajes básicos, que serán fundamentales durante el resto de la vida. Los niños del campo o los actores de la población rural, si necesitan esa prioridad.

Al fortalecer la administración local, se generan condiciones de igualdad más duraderas en el largo plazo, incidiendo esto en la segregación social, económica y espacial. Al existir una administración fortalecida $y$ confiable, crecen las garantías para la población y disminuye la corrupción.

\section{Conclusiones}

El DEH y los EO pueden relacionarse entre sí para realizar evaluaciones a PD alternativas y complementarias que permitan identificar los efectos de las acciones llevadas a cabo y su 
relación con las necesidades humanas básicas fundamentales.

La unión entre las EO y DEH permite identificar las incidencias de los satisfactores acordados en los PD identificando la dinámica del satisfactor según los resultados obtenidos y validados en el territorio. Simultáneamente permite identificar las necesidades que quedaron sin satisfacer.

Los resultados obtenidos en la evaluación de EO, indican que el efecto de segundo grado con más probabilidad de ocurrencia es el de la Segregación social, económica, espacial y cultural. Seguido por, Salud como derecho fundamental y en tercer lugar por la dependencia económica y social. Lo que indica que los incentivos del PD local de Usme, efectivamente impactan la segregación dentro del territorio evaluado como se pretendía desde el Acuerdo Local.
Se observó que el PD se enfocó mayoritariamente en la Participación desde el hacer, mientras se dejó sin satisfacer las necesidades del Afecto y del Ocio.

Una vez realizada la evaluación se pudo identificar el tipo de satisfactor a partir de las incidencias de las acciones en el territorio validadas con los participantes en la investigación. Dando como resultado que de los 8 incentivos/satisfactores evaluados, 4 fueron sinérgicos, 1 singular, 1 Pseudo-Satisfactor, 1 Destructor y 1 Inhibidor.

Se demostró que los fenómenos locales con altos grados complejidad como las incidencias de los PD dentro de un territorio, pueden ser evaluados de una manera alternativa y colaborativa sin ser deformados, simplificados o separados de la realidad. 
Efectos olvidados y desarrollo a escala humana, una propuesta alternativa para la evaluación...

\section{Referencias}

Abarca, A. (2002). Las políticas públicas como perspectiva de análisis. Ciencias Sociales, 97(III),95-103.

Alcaldía Mayor. (2011). Exposición de motivos. Disponible en: http://www.sdp.gov.co/portal/page/portal/PortalSDP/Home/Noticias/OtrosDocumentosArchiva dos/PlandeDesarrollo/Exposicion_Motivos_Plan_Bogota_Humana_20120430.pdf . Acceso en 21 de enero. 2018.

Concejo de Bogotá. (2012). Plan de desarrollo 2012-2016. Disponible en: http://www.sdp.gov.co/portal/page/portal/PortalSDP/Home/Noticias/OtrosDocumentosArchiv ados/PlandeDesarrollo/Acuerdo_489_2012_PDD.pdf.

Cruz, I., Atahel, A., y Max-Neef, M. (2009). Towards a Systematic Development Approach: Building on the Human-Scale Development Paradigm. Ecological Economics, 68(7), 2021-2030.

Cunningham, W. P. y Saigo, B. W. (2001). Environmental Science: A global concern. Boston: McGraw-Hill.

Ducey, M. y Larson, B. (1999). A fuzzy set approach to the problem of sustainability. Forest Ecology and Management, 115(1), pp. 29-40.

Flores, R. (2009). La entrevista como técnica de investigación social. En R. Flores, Observando observadores: Una Introducción a las técnicas cualitativas. Santiago: Universidad Católica de Chile.

Gil-Lafuente, A. y Kyun, Y. (2012). Incidencia de las inversiones de la administración pública sobre el desarrollo turístico de una ciudad. Revista Venezolana de Gestión Pública, 3(3), pp. 81-109.

Guba, E., y Lincoln, Y. (2002). Paradigmas en competencia en investigación cualitativa. En C. Denham, y J. Haro, Por los rincones. Antología de métodos cualitativos en la investigación social. Región y sociedad, 14(23), 364.

Jiménez, J. \& Podestá, A. (2009). Inversión, incentivos fiscales y gastos tributarios en América Latina. Publicación de las naciones Unidas. Santiago de Chile.

Junta Administradora local de Usme. Acuerdo Local 002 de 2012. Disponible en: http://www.alcaldiabogota.gov.co/sisjur/normas/Norma1.jsp?i=50127 Acceso en 21 de enero. 2018.

Kaufmann, A. y Gil-Aluja, J. (1986). Introducción de la teoría de los subconjuntos borrosos a la gestión de las empresas. Santiago de Compostela: Milladoiro.

- (1988). Técnicas operativas de gestión para el tratamiento de la incertidumbre. Santiago de Compostela: Hispano Europea.

Manna, E. M., Rojas, J., y Mondaca C. (2018). Application of the Forgotten Effects Theory for Assessing the Public Policy on Air Pollution of the Commune of Valdivia, Chile, Springer, 61-72.

Max-Neef, M., Elizalde, A. y Hopenhayn, M. (1986). Desarrollo a escala humana Opciones para el futuro. Santiago, Chile: CEPAUR.

Muñoz, M. J., Rivera, J. M. y Moneva, J. M. (2008). Evaluating sustainability in organisations with a fuzzy logic approach. Industrial Management \& Data Systems, 108(6), pp. 829-841.

Phillis, Y. y Andriantiatsaholiaina, L. (2001). Sustainability: an ill-defined concept and its assessment using fuzzy logic. Ecological Economics, 37(3), pp. 435-56.

Pinzon, F. (2015). Escapadas por Colombia. Bogotá: El Tiempo.

Rittel, H. y Webber, M. (1973). Dilemmas in a general theory of planning. Policy Sciences, 4(2), pp. 155-169.

Sánchez, L. y Ramírez, J. (2010). Propuesta de un modelo para la evaluación de impactos de programas sociales desde las teorías de desarrollo a escala humana y las dimensiones de la sostenibilidad. Foro virtual de contabilidad ambiental y social.

Secretaría Distrital de Planeación. (2011). Guía para la formulación ejecución y evaluación de los planes de desarrollo distrital y local. Disponible http://www.sdp.gov.co/portal/page/portal/PortalSDP/Home/Noticias/OtrosDocumentosArchiv ados/GuiaPlanesDesarrollo/Guia_formulacion_PDD_PDL.pdf. 